\title{
Characterisation and Clinical Significance of FLT3-ITD and non-ITD in Acute Myeloid Leukaemia Patients in Kelantan, Northeast Peninsular Malaysia
}

\author{
Noraini Mat Yunus ${ }^{1}$, Muhammad Farid Johan ${ }^{1 *}$, Hamid Ali Nagi Al-Jamal', \\ Azlan Husin ${ }^{2}$, Abdul Rahim Hussein ${ }^{3}$, Rosline Hassan ${ }^{1}$
}

\begin{abstract}
Background: Mutations of the FMS-like tyrosine kinase-3 (FLT3) receptor gene may promote proliferation via activation of multiple signaling pathways. FLT3-internal tandem duplication (FLT3-ITD) is the most common gene alteration found in patients diagnosed with acute myeloid leukaemia (AML) and has been associated with poor prognosis. Materials and Methods: We performed mutational analysis of exons 14-15 and 20 of the FLT3 gene in 54 AML patients using PCR-CSGE (conformational sensitive gel electrophoresis) followed by sequencing analysis to characterise FLT3 mutations in adult patients diagnosed with AML at Hospital USM, Kelantan, Northeast Peninsular Malaysia. Results: FLT3 exon 14-15 mutations were identified in 7 of 54 patients (13\%) whereas no mutation was found in FLT3 exon 20. Six ITDs and one non-ITD mutation were found in exon 14 of the juxtamembrane (JM) domain of FLT3. FLT3-ITD mutations were associated with a significantly higher blast percentage $(p-v a l u e=0.008)$ and white blood cell count $(p$-value $=0.023)$ but there was no significant difference

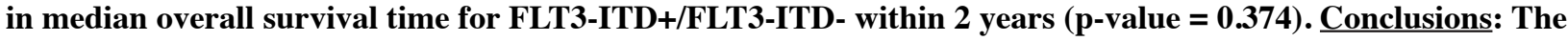
incidence of FLT3-ITD in AML patients in this particular region of Malaysia is low compared to the Western world and has a significant association with WBC and blast percentage.
\end{abstract}

Keywords: AML - FLT3 - ITD - CSGE

Asian Pac J Cancer Prev, 16 (12), 4869-4872

\section{Introduction}

FLT3 mutations are the most frequent identified genetic abnormalities in patients diagnosed with acute myeloid leukaemia (AML) (Small, 2006). Two types of FLT3 mutations have been identified; internal tandem duplications (FLT3-ITD) in juxtamembrane domain (JM) and point mutations in tyrosine kinase domain (FLT3TKD) (Kiyoi et al., 2002; Meshinchi et al., 2008). The length of the ITD mutation varies from 3-400bp nucleotides (Stirewalt and Radich, 2003). FLT3-ITD accounted in 25\% for adult (Frohling et al., 2002; Schnittger et al., 2002; Thiede et al., 2002). Another common type of FLT3 mutation is a point mutation at codon D835 or I836 in exon 20 within the activation loop of TKD. FLT3-TKD is less frequently seen in AML and they account for $5 \%$ of adult (Abu-Duhier et al., 2001; Yamamoto et al., 2001). Both FLT3-ITD and FLT3-TKD resulting in ligand independent and leads to continuous activation of FLT3 receptor (Betz and Hess, 2010; Gulley et al., 2010). FLT3-ITD in AML patients confer poor prognosis; in which it is associated with high percentage of blast cells, increased risk of relapse from complete remission, and reduced survival (Kottaridis et al., 2001; Frohling et al., 2002; Small, 2006; Karabacak et al., 2010; Feng et al., 2012; Zeichner et al., 2014). However, the prognosis of FLT3-TKD in AML is still unclear (Whitman et al., 2010; Smith et al., 2011). The high incidence of FLT3 mutations as well as the poor prognosis status in AML eventually drew an attention towards the development of specific treatment for AML through FLT3 aberration. So far, the incidence of FLT3 mutations in Malaysia still limited and to our knowledge this is the first study that detects the incidence of non-ITD mutations and their correlation with clinical parameters compared to FLT3-ITD mutations in patients diagnosed with AML at Hospital USM.

\section{Materials and Methods}

\section{Patients and controls}

Bone marrow aspiration and peripheral blood samples from 54 adults with AML (16-76 y) were collected at diagnosis from Hospital USM between 2009 and 2012. Genomic DNA was obtained at presentation classified

${ }^{1}$ Department of Hematology, ${ }^{2}$ Haemato-Oncology Unit, Department of Internal Medicine, School of Medical Sciences, Universiti Sains Malaysia, Kubang Kerian, Kelantan, ${ }^{3}$ Advanced Medical And Dental Institute, Universiti Sains Malaysia, Bertam, Kepala Batas, Pulau Pinang, Malaysia*For correspondence:faridjohan@usm.my 
according to the French-American-British (FAB) criteria as: M0 ( $n=1)$, M1 ( $=2)$, M2 ( $=8)$, M3 ( $=18)$, M4 ( $=7)$, M5 $(n=11)$, M6 $(n=2)$, M7 $(n=1)$ and four undefined. A thirty normal control peripheral blood samples were collected from screened healthy blood donors from Blood Transfusion Unit, Hospital USM, following informed consent. This study was approved by the Human Research Ethics Committee, Universiti Sains Malaysia (FWA Reg. No: 00007718; IRB Reg. No: 00004494).

\section{DNA amplification}

Genomic DNA was harvested using DNA extraction kit, Promega Wizard (Promega, USA) and amplified by polymerase chain reaction (PCR). Amplification of FLT3ITD exon 14-15 and FLT3-TKD exon 20 were carried out using primers as shown in Table 1 . The total reaction volume of $25 \mu \mathrm{L}$ contained approximately $50 \mathrm{ng} / \mathrm{ul}$ DNA, $10 \mu \mathrm{M} / \mu \mathrm{L}$ of each primer and 1.1X Promega Green master mix (Promega, USA). Samples were amplified by thermal cycler (Applied Biosystem, USA) using the following conditions: $95^{\circ} \mathrm{C}$ for 2 minutes; 35 cycles of $95^{\circ} \mathrm{C}$ for 30 seconds; FLT3-ITD $52^{\circ} \mathrm{C}$, FLT3-TKD $55^{\circ} \mathrm{C}$ for 35 minute; $72^{\circ} \mathrm{C}$ for 65 seconds and finally $72^{\circ} \mathrm{C}$ for 5 minutes. PCR products were visualised by agarose gel electrophoresis with 100 bp DNA ladder (Promega, USA) to confirm the amplification of fragment of FLT3-ITD and FLT3-TKD.

\section{DNA mutation analysis}

Mutation screening was done by Conformation Sensitive Gel Electrophoresis (CSGE) analysis. To enable heteroduplex formation for CSGE analysis, PCR products were denatured at $98^{\circ} \mathrm{C}$ for 5 minute and then re-annealed at $65^{\circ} \mathrm{C}$ for 30 minute. A 41x33x0.1 cm gel was prepared with $10 \%$ polyacrylamide and a $99: 1$ ratio acrylamide to bisacryloylpiperazin (BAP), 10\% ethylene glycol, 15\% formamide in 0.5X Tris-Taurine-EDTA (TTE) buffer, $1.75 \mathrm{~mL} 10 \%$ Ammonium Persulfate (APS) and $100 \mu \mathrm{L}$ Tetramethylethylenediamine (TEMED) and loaded with $4 \mu \mathrm{L}$ heteroduplexed PCR products. The gel was electrophoresed at $400 \mathrm{~V}$ for 16 hours at room temperature, post-stained in Ethidium Bromide and visualised under UV light. Samples displaying abnormal CSGE profiles compared to that obtained from a normal individual were directly sequenced.

\section{Statistical analysis}

Fisher's exact test was employed to compare the categorical variables between ITD and non-ITD mutations and Mann-Whitney test was performed to determine the differences of continuous variables between both groups. Survival was analysed according to the Kaplan-Meier method. All statistical analyses were performed using the SPSS software package (Version 20, SPSS, Armonk, NY, USA) and the level of significant was considered at $\mathrm{p}<0.05$.

\section{Results}

Alteration of FLT3 exon 14-15 was identified in 7 cases of 54 adult AML patients (13\%). Six cases were shown to have internal tandem duplication (ITD) mutation ranging from 15 to 66 base pairs (bp) inframe duplication and they were unique in each case. Only one case was detected to have a non-ITD mutation (case 49) involved in FLT3/1415 and it was a deletion of $\mathrm{C}$ nucleotide at position 1751 (Figure 1). Case 49 was a 50 years old woman with transition-AML NOS subtype and died within two weeks after diagnosed as AML. She suffered from breast cancer for four years before diagnosed with transition-AML. Other six cases with ITD were de novo AML. None of the 54 patients displayed FLT3-TKD mutation.

The clinical characteristics of 54 AML patients with or without FLT3-ITD mutation are described in Table 2. There were more females among AML patients with FLT3-ITD mutation. The presence of FLT3-ITD mutation significantly correlated to the higher level of white blood cell count and higher blast percentage.

Forty-eight patients had a complete survival data available for analysis of FLT3-ITD+ (mutant) and FLT3ITD- (wild type) classification as presented in Table 3

Table 1. Primers used in Amplification of FLT3 Genes

\begin{tabular}{llc}
\hline Gene & \multicolumn{1}{c}{$\begin{array}{c}\text { Primer sequences } \\
\left(5^{\prime}-3^{\prime}\right)\end{array}$} & $\begin{array}{c}\text { Fragment } \\
\text { size (bp) }\end{array}$ \\
\hline FLT3-ITD & $\begin{array}{l}\text { forward: gcaatttaggtatgaaagccagc } \\
\text { reverse: ctttcagcatttgacggcaacc } \\
\text { forward: cctactgaagttgagtctag } \\
\text { FLT3-TKD }\end{array}$ & 329 \\
& \begin{tabular}{l} 
reverse: gtgagtgcagttgtttacca \\
\hline
\end{tabular} & \\
\hline
\end{tabular}

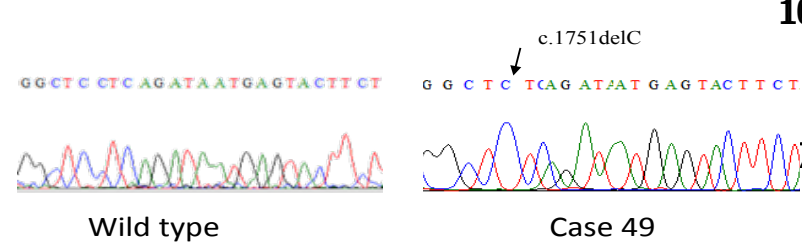

Figure 1. C Nucleotide Deletion at Position 1751 in Non-ITD

Table 2. Clinical Data of AML 3 Patients with and without FLT3-ITD

\begin{tabular}{lccc}
\hline Parameters & FLT3-ITD & FLT3 wild type & p-value \\
\hline Gender (Male/Female) & $2 / 4$ & $18 / 29$ & $1.000^{\mathrm{a}}$ \\
Median age (year) & $41-50$ & $31-40$ & $0.703^{\mathrm{a}}$ \\
Median WBC (x10/L) & 61 & 13 & $0.023^{\mathrm{b}}$ \\
Median Hb (g/dL) & 7.3 & 7.9 & $0.129^{\mathrm{b}}$ \\
Median Plt (x10 $/ \mathrm{L})$ & 30 & 34 & $0.705^{\mathrm{b}}$ \\
Median blast & 88 & 46 & $0.008^{\mathrm{b}}$ \\
\hline
\end{tabular}

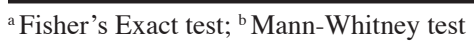

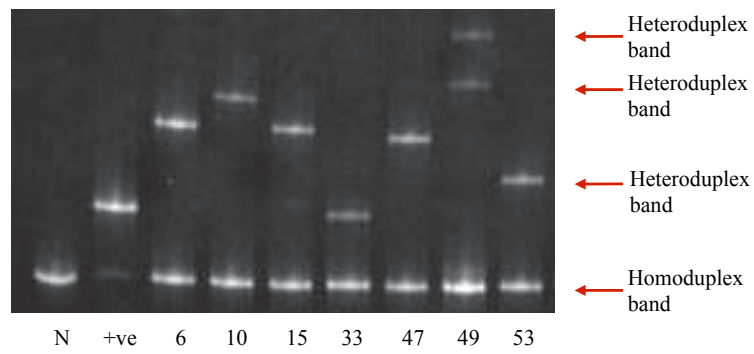

Figure 2. CSGE Analysis of FLT3-ITD. Multiple band aberrations seen on CSGE gel for cases 6, 10, 15, 33, 47, 49 and 53 are related to heteroduplex formation between wildtype and mutant alleles of FLT3-ITD gene. N: normal control, +ve: positive control for FLT3-ITD (MV4-11 cell line), 6/10/15/33/47/49/53: cases 
Table 3. Differences of Median Survival Time of AML Patients with and without FLT3-ITD

\begin{tabular}{lrrccc}
\hline FLT3-ITD & $\mathrm{n}$ & Survive $(\%)$ & Median $(95 \%$ CI) (weeks) & Log Ranka (df) & p-value \\
\hline Mutation & 6 & $2(33.3)$ & $9.00(0.00,84.62)$ & $0.194(1)$ & 0.660 \\
No mutation & 42 & $16(38.1)$ & $52.00(8.60,95.40)$ & & \\
\hline
\end{tabular}

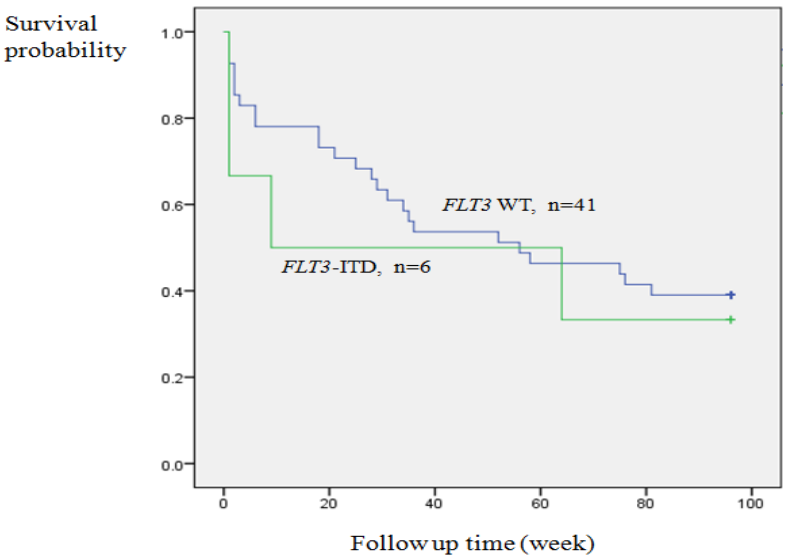

Figure 3. Survival Analysis of 48 AML Patients Carrying Mutant FLT3-ITD and Wild Type FLT3. Forty-seven patients had a complete survival data available for analysis of FLT3-ITD+ (mutant) and FLT3-ITD- (wild type) classification. Patients without FLT3-ITD have longer median survival time (52 weeks) compared to patients who have FLT3ITD (9 weeks)

$\begin{array}{lc}\text { WT } & \text { QFRYESQLQMVQVTGSSDNEYFYVDFREYEYDLKWEFPRENLEF } \\ 6 & \text { EYFYVDFREYEYDLKWEFP } \\ 10 & \text { DNEYF } \\ 15 & \text { EYFYVDFREYEYDLKWEFPREN } \\ 33 & \text { YVDFPEYEYDLEWKFPR } \\ 47 & \text { DFI EYEYDLKWEFPRE } \\ 53 & \text { VTGSSDNEYFYVDFREYE }\end{array}$

Figure 4. Sites of ITD in Exon 14 of FLT3 Gene. The underlined amino acids represent amino acids substitution within ITD site. WT: wild type, 6/10/15/33/47/53: cases

and Figure 3. Patients without FLT3-ITD have longer median survival time (52 weeks) compared to patients with FLT3-ITD (9 weeks). However, statistically, there was no significant difference of median two years survival time for FLT3-ITD+ and FLT3-ITD- (p-value = 0.374).

\section{Discussion}

Mutations of tyrosine kinase enhance cell proliferation and development of haematologic malignancies (Liang et al., 2003). The results of this study revealed mutations within JM domain in 7 cases of 54 (13\%) AML patients and six of them were FLT3-ITD, however there was no mutation identified in TKD. These findings are in accordance to that previously reported by Karabacak et al. (2010). In addition, the results revealed that FLT3-ITD do not show any specific correlation to a particular AML FAB subtype, but they were more frequently found in AML-M3 (APML) cases compared to non APML cases which is consistent with previous reports (Schnittger et al., 2002; Thiede et al., 2002).

ITDs always led to an in-frame transcription, the mutant ITD sequences and length were unique in each case, with addition of repeated nucleotides ranging from 15 to 66 bases and 5 to 22 amino acids. All of the ITD cases were only restricted in JM within exon 14 of the FLT3 receptor. The starting and ending for nucleotide and amino acid positions were unique in every case. Two ITD cases (33 and 47) demonstrated amino acid substitutions and manifested at the same location of ITD site but different amino acids substitutions (Figure 4). These results are in agreement with previous reports (Stirewalt and Radich, 2003; Ahmad et al., 2010). One of the FLT3 mutations within exon 14 (case 49) was not an internal repetition of the nucleotides or amino acids but a one-nucleotide deletion. This mutation also contributed to the frame shift nucleotide sequences and resulted in a shorter protein production for FLT3 as the stop codon TAG was translated at codon 610 .

FLT3 mutations cause FLT3 tyrosine kinase activation resulting in constitutive activation of pathways leading to uncontrolled proliferation and lower apoptosis (Stirewalt and Radich, 2003; Karabacak et al., 2010). Therefore, it was our interest to look for any correlation of FLT3 mutations with clinical parameters (Table 2). The clinical data analysis showed no correlation between age, gender, hemoglobin level and platelet count with mutant FLT3 compared to wild type FLT3. On the other hand, the results revealed significant higher leukocytosis in patients with FLT3-ITD $(\mathrm{p}=0.023)$ and higher blast percentage $(\mathrm{p}=0.008)$ compared to patients with wild-type FLT3. These findings are in accordance to previously reported (Kottaridis et al., 2001; Frohling et al., 2002; Wang et al., 2010; Su et al., 2013; Elyamany et al., 2014) but in disagreement with that reported by Shahab et al. (2013).

In conclusions, FLT3-ITD mutations are less frequent in adult Malaysian AML patients compared to the other countries, with no mutation found in TKD of FLT3. ITD and Non-ITD types of mutation were unique in each case of Malaysian AML. For further study, a larger Malaysian AML population is needed in order to analyse the pattern of FLT3 mutation as well as determination of prognostic impact of FLT3-ITD in Malaysian AML population.

\section{Acknowledgements}

This work was supported by a short term grant (304/ PPSP/61312004) from Universiti Sains Malaysia to MFJ. We thank all staffs at Central Research Laboratory and Molecular Haematology Laboratory, School of Medical Sciences and Craniofacial Laboratory, School of Dental Sciences, Universiti Sains Malaysia who provided laboratory supports.

\section{References}

Abu-Duhier FM, Goodeve AC, Wilson GA, et al (2001). Identification of novel FLT-3 Asp835 mutations in adult 
Noraini Mat Yunus et al acute myeloid leukaemia. Br J Haematol, 113, 983-8.

Ahmad F, Mandava S, Das BR (2010). Analysis of FLT3-ITD and FLT3-Asp835 mutations in de novo acute myeloid leukemia: evaluation of incidence, distribution pattern, correlation with cytogenetics and characterization of internal tandem duplication from Indian population. Cancer Invest, 28, 63-73.

Betz BL, Hess JL (2010). Acute myeloid leukemia diagnosis in the 21st century. Arch Pathol Lab Med, 134, 1427-33.

Elyamany G, Awad M, Fadalla K, et al (2014). Frequency and prognostic relevance of FLT3 mutations in saudi acute myeloid leukemia patients. Adv Hematol, 2014, 141360.

Feng JH, Guo XP, Chen YY, et al (2012). Prognostic significance of IDH1 mutations in acute myeloid leukemia: a metaanalysis. Am J Blood Res, 2, 254-64.

Frohling S, Schlenk RF, Breitruck J, et al (2002). Prognostic significance of activating FLT3 mutations in younger adults (16 to 60 years) with acute myeloid leukemia and normal cytogenetics: a study of the AML Study Group Ulm. Blood, 100, 4372-80.

Gulley ML, Shea TC, Fedoriw Y (2010). Genetic tests to evaluate prognosis and predict therapeutic response in acute myeloid leukemia. J Mol Diagn, 12, 3-16.

Karabacak BH, Erbey F, Bayram I, et al (2010). Fms-like tyrosine kinase 3 mutations in childhood acute leukemias and their association with prognosis. Asian Pac J Cancer Prev, 11, 923-7.

Kiyoi H, Ohno R, Ueda R, et al (2002). Mechanism of constitutive activation of FLT3 with internal tandem duplication in the juxtamembrane domain. Oncogene, 21, 2555-63.

Kottaridis PD, Gale RE, Frew ME, et al (2001). The presence of a FLT3 internal tandem duplication in patients with acute myeloid leukemia (AML) adds important prognostic information to cytogenetic risk group and response to the first cycle of chemotherapy: analysis of 854 patients from the United Kingdom Medical Research Council AML 10 and 12 trials. Blood, 98, 1752-9.

Liang DC, Shih LY, Hung IJ, et al (2003). FLT3-TKD mutation in childhood acute myeloid leukemia. Leukemia , 17, 883-6.

Meshinchi S, Stirewalt DL, Alonzo TA, et al (2008). Structural and numerical variation of FLT3/ITD in pediatric AML. Blood, 111, 4930-3.

Schnittger S, Schoch C, Dugas M, et al (2002). Analysis of FLT3 length mutations in 1003 patients with acute myeloid leukemia: correlation to cytogenetics, FAB subtype, and prognosis in the AMLCG study and usefulness as a marker for the detection of minimal residual disease. Blood, 100, 59-66.

Shahab S, Shamsi TS, Ahmed N (2013). Prognostic involvement of nucleophosmin mutations in acute myeloid leaukemia. Asian Pac J Cancer Prev, 14, 5615-20.

Small D (2006). FLT3 mutations: biology and treatment. Hematology Am Soc Hematol Educ Program, 178-84.

Smith ML, Hills RK, Grimwade D (2011). Independent prognostic variables in acute myeloid leukaemia. Blood Rev, 25, 39-51.

Stirewalt DL, Radich JP (2003). The role of FLT3 in haematopoietic malignancies. Nat Rev Cancer, 3, 650-65.

Su L, Gao SJ, Tan YH, et al (2013). Associations between age, cytogenetics, FLT3-ITD, and marrow leukemia cells identified by flow cytometry. Asian Pac J Cancer Prev, 14, 5341-4.

Thiede C, Steudel C, Mohr B, et al (2002). Analysis of FLT3activating mutations in 979 patients with acute myelogenous leukemia: association with FAB subtypes and identification of subgroups with poor prognosis. Blood, 99, 4326-35.

Wang L, Xu WL, Meng HT, et al (2010). FLT3 and NPM1 mutations in Chinese patients with acute myeloid leukemia and normal cytogenetics. J Zhejiang Univ Sci B, 11, 762-70.

Whitman SP, Maharry K, Radmacher MD, et al (2010). FLT3 internal tandem duplication associates with adverse outcome and gene- and microRNA-expression signatures in patients 60 years of age or older with primary cytogenetically normal acute myeloid leukemia: a Cancer and Leukemia Group B study. Blood, 116, 3622-6.

Yamamoto Y, Kiyoi H, Nakano Y, et al (2001). Activating mutation of D835 within the activation loop of FLT3 in human hematologic malignancies. Blood, 97, 2434-9.

Zeichner SB, Alghamdi S, Elhammady G, et al (2014). Prognostic significance of TP53 mutations and single nucleotide polymorphisms in acute myeloid leukemia: a case series and literature review. Asian Pac J Cancer Prev, 15, 1603-9. 